\title{
Genetic mapping of sex determination in a wild strawberry, Fragaria virginiana, reveals earliest form of sex chromosome
}

\author{
RB Spigler ${ }^{1}$, KS Lewers ${ }^{2}$, DS Main ${ }^{3}$ and T-L Ashman ${ }^{1}$ \\ ${ }^{1}$ Department of Biological Sciences, University of Pittsburgh, Pittsburgh, PA, USA; ${ }^{2}$ Genetic Improvement of Fruits and Vegetables Lab, \\ USDA-ARS, Beltsville Agricultural Research Center, Beltsville, MD, USA and ${ }^{3}$ Department of Horticulture and Landscape \\ Architecture, Washington State University, Pullman, WA, USA
}

\begin{abstract}
The evolution of separate sexes (dioecy) from hermaphroditism is one of the major evolutionary transitions in plants, and this transition can be accompanied by the development of sex chromosomes. Studies in species with intermediate sexual systems are providing unprecedented insight into the initial stages of sex chromosome evolution. Here, we describe the genetic mechanism of sex determination in the octoploid, subdioecious wild strawberry, Fragaria virginiana Mill., based on a whole-genome simple sequence repeat (SSR)-based genetic map and on mapping sex determination as two qualitative traits, male and female function. The resultant total map length is $2373 \mathrm{~cm}$ and includes 212 markers on 42 linkage groups (mean marker spacing: $14 \mathrm{~cm}$ ). We estimated that approximately 70 and $90 \%$ of the total $F$. virginiana genetic map resides within 10 and $20 \mathrm{~cm}$ of a
\end{abstract}

marker on this map, respectively. Both sex expression traits mapped to the same linkage group, separated by approximately $6 \mathrm{cM}$, along with two SSR markers. Together, our phenotypic and genetic mapping results support a model of gender determination in subdioecious $F$. virginiana with at least two linked loci (or gene regions) with major effects. Reconstruction of parental genotypes at these loci reveals that both female and hermaphrodite heterogamety exist in this species. Evidence of recombination between the sexdetermining loci, an important hallmark of incipient sex chromosomes, suggest that $F$. virginiana is an example of the youngest sex chromosome in plants and thus a novel model system for the study of sex chromosome evolution. Heredity (2008) 101, 507-517; doi:10.1038/hdy.2008.100; published online 17 September 2008

Keywords: dioecy; Fragaria; gynodioecy; male sterility; sex chromosome; sex linkage

\section{Introduction}

Although the presence of separate sexes (that is, dioecy) is common in animals, it is relatively rare in plants (Renner and Ricklefs, 1995). However, separate sexes have evolved from hermaphroditism numerous times and through numerous pathways in angiosperms (Charlesworth, 1999). One particularly well-studied pathway involves an intermediate sexual system known as gynodioecy, and this pathway occurs in two steps. In the first step, male sterile mutants (that is, females) invade and are maintained among hermaphrodites (gynodioecy). In the second step, female sterile mutants (that is, males) invade (subdioecy) and ultimately supplant hermaphrodites (dioecy) (Charlesworth, 1999). Species with intermediate sexual systems, such as gynodioecy and subdioecy, have proven to be powerful systems for understanding the evolution of sexual

Correspondence: Dr T-L Ashman, Department of Biological Sciences, University of Pittsburgh, Pittsburgh, PA 15260-3929, USA.

E-mail: tia1@pitt.edu or Dr KS Lewers, Genetic Improvement of Fruits and Vegetables Lab, USDA-ARS, Beltsville Agricultural Research Center, Beltsville, MD 20705-2350, USA.

E-mail:kim.lewers@ars.usda.gov

Received 1 May 2008; revised 25 July 2008; accepted 8 August 2008; published online 17 September 2008 dimorphism as well as the genetic underpinnings of sexual system evolution (reviewed by Ashman, 2003).

The evolution of sex chromosomes is also thought to be a stepwise process. The process is initiated by male and female sterility mutations that arise in the progenitor hermaphrodite genome, followed by selection for linkage between the male- and female-function genes, and ultimately, suppression of recombination between them (Charlesworth and Charlesworth, 1978; Charlesworth and Guttman, 1999; Charlesworth et al., 2005). After these events, one chromosome would carry the male sterility allele that produces females and the other chromosome would carry the female sterility allele that produces males. When the female sterility allele is dominant, and suppression between the sex-determining loci has occurred, males are the heterogametic sex, as found in several dioecious species (Charlesworth and Charlesworth, 1978; Charlesworth and Guttman, 1999). However, when the male sterility allele is dominant, females can become the heterogametic sex, a less common condition in plants (Charlesworth and Charlesworth, 1978; Vyskot and Hobza, 2004). Further modifications of the sex chromosomes are expected to lead to the accumulation of genes with sex-specific function by sexually antagonistic selection (Rice, 1984).

Given the putatively gradual nature of sex chromosome evolution, study in plant systems without strict 
dioecy and without heteromorphic sex chromosomes should help in deciphering the process of sex chromosome evolution (Charlesworth et al., 2005; Ming et al., 2007). For example, genetic mapping in trioecious papaya and dioecious asparagus have revealed single sex-determining regions in each that have a degree of recombination suppression (Liu et al., 2004; TelgmannRauber et al., 2007), indicating early steps in sex chromosome evolution. Genetic mapping of sex determination in plant species with intermediate sexual systems, such as gynodioecy or subdioecy, thus ought to provide novel insight into the very earliest stages of sex chromosome evolution in plants. Yet, to the best of our knowledge, these studies do not exist.

The octoploid Virginian wild strawberry, Fragaria virginiana Mill. (Rosaceae), provides a prime opportunity in this regard because it has a gynodioecious to subdioecious sexual system and has hermaphrodite progenitors (F. vesca and F. nubicola) plus a fully dioecious sibling species (F. chiloensis) (Potter et al., 2000). It is believed to be an alloallopolyploid ( $\mathrm{AAA}^{\prime} \mathrm{A}^{\prime} \mathrm{BBB}^{\prime} \mathrm{B}^{\prime}$; Bringhurst, 1990) with disomic inheritance $(2 n=8 \times=56)$ (Ashley et al., 2003). Moreover, there is a long history of interest in sex determination in the wild octoploid Fragaria species (F. virginiana and F. chiloensis), because they are the progenitors of the cultivated strawberry, $F$. $\times$ ananassa, and continue to be a significant source of germplasm for crop improvement (for example, Maas et al., 2002).

In $F$. virginiana, females coexist with pollen-bearing morphs (hermaphrodites and/or males). Females can be easily identified by a complete lack of pollen production and consistently high fruit set (Ashman, 2003). Pollenbearing morphs vary in their ability to set fruit and thus have been difficult to categorize. In fact, these have been referred to in several different ways in the literature (Valleau, 1923; Hancock and Bringhurst, 1979; Stahler et al., 1995). This variation in fruit setting ability of F. virginiana pollen-bearing morphs is not surprising given that wild populations vary widely in the frequency of females (Stahler et al., 1995; Ashman, 1999) and, according to evolutionary theory, female frequency ought to impose selection on hermaphrodite sexual expression (reviewed by Ashman, 2006). Indeed, populations with high frequencies of females have hermaphrodites with low or no fruit setting ability and vice versa (Ashman, 1999).

Previous classical genetic work in the sexually dimorphic octoploid strawberries ( $F$. virginiana and $F$. chiloensis) concluded that sex is determined by a disomically inherited single nuclear locus with the three alleles ' $F$ ', ' $m$ ' and ' $h$ ' (Valleau, 1923; Ahmadi and Bringhurst, 1989). According to this model, a male sterility allele (' $F$ ' for femaleness) suppresses anther and/or pollen development and is dominant over a female sterility allele (' $\mathrm{m}$ ' for maleness), and over an allele that confers hermaphroditism $\left({ }^{\prime} \mathrm{h}\right.$ '), which in turn is dominant over ' $\mathrm{m}$ ' (Ahmadi and Bringhurst, 1989). Thus, in this model, females are always heterogametic ' $\mathrm{Fh}^{\prime}$ or 'Fm,' whereas males are ' $\mathrm{mm}$ ' and hermaphrodites are either ' $\mathrm{hh}$ ' or ' $\mathrm{hm}$ ' (Ahmadi and Bringhurst, 1989).

However, several lines of evidence suggest that the single gene theory of sex determination is an oversimplification. First, clones of $F$. virginiana and $F . \times$ ananassa that are both female and male sterile (that is, neuter) have been found in the wild and in cultivation (Valleau, 1923; Bushakra, pers. com; Ashman, pers. obs.), and high-fruiting hermaphrodites can occur in dioecious populations of $F$. chiloensis in California, USA (Hancock and Bringhurst, 1979). Both of these phenotypes could indicate recombination between two or more closely linked loci rather than a single locus with three alleles (Valleau, 1923). In fact, some early study concluded that as many as four loci may exist in tetraploid and eight in octoploid strawberries (Staudt, 1967). Second, genes of additive effect also play a role, together with the plant's environment, in regulating quantitative variation in sex expression (Valleau, 1923; Stahler et al., 1995; Ashman, 1999, 2003). Genetic mapping of sex determination can resolve these conflicting findings and lead to a better understanding of sex determination in this group. Indeed, recent genetic mapping efforts in other gender dimorphic plant species have provided insight into the structure and nature of sex determination mechanisms (for example, Liu et al., 2004; Telgmann-Rauber et al., 2007; Yin et al., 2008).

Although genetic maps exist for a diploid wild strawberry interspecific cross, F. vesca $\times F$. nubicola, (Sargent et al., 2007) and octoploid cultivated strawberry F. $\times$ ananassa (Lerceteau-Köhler et al., 2003; Weebadde et al., 2008), these species do not show sex dimorphism and cannot shed light on the question of dimorphic sex determination. Thus, to gain insight into the genetic mechanism of sex determination in the octoploid strawberry F. virginiana, we created an simple sequence repeat (SSR)-based whole-genome genetic map and mapped sex determination as two qualitative traits, male and female function.

\section{Methods}

Source of material and mapping population cultivation We created a $F$. virginiana mapping population from an inter-population cross with a female maternal parent and a hermaphrodite paternal parent. The parents were chosen to capture the greatest diversity of putative sexdetermining genes/alleles on the basis of test crosses. The maternal parent (Y33b2) was the product of a cross between plants originating in a wild population in northwest Pennsylvania (PA) that has a high proportion of females (population W, by Ashman, 1999). Y33b2's hermaphrodite full-siblings often set no fruit (unpublished data), and test crosses revealed that progeny of Y33b2 segregated for fruit setting ability as well. The paternal parent (O477) was originally collected from a different northwest PA population with a medium frequency of females (population PR, by Ashman, 1999). O477 had a low-to-moderate level of fruit set (mean of $17 \%$ when tested under a range of growth conditions), and test crosses with self-pollen revealed segregation for fruit setting ability in the progeny (unpublished data). Following the model of Ahmadi and Bringhurst (1989), the female parent genotype was assumed to be ' $\mathrm{Fm}$ ', and the hermaphrodite ' $\mathrm{hm}$ '.

Flowers on $\mathrm{Y} 33 \mathrm{~b} 2$ were pollinated by hand with pollen collected from O477 in May 2005, and the resultant seeds were harvested and stored at $-20^{\circ} \mathrm{C}$. In February 2006, we planted 300 seeds and then randomly chose 184 seedlings, which we then repotted. We produced six clonal replicate plants from each progeny and the two parents in the greenhouse at the University of Pittsburgh 
(UPitt). These clones were divided into two groups and grown in a randomized block design at each of two locations (UPitt and USDA-ARS Beltsville, MD, USA). In the greenhouse at UPitt, three clones of each progeny were grown in $200 \mathrm{ml}$ pots filled with a 1:2 sand and Fafard no. 4 soil mix and were given 10 beads of Nutricote 13:13:13 N:P:K fertilizer. Water and pest control were given as needed. In the field at USDA-ARS, three clones of each progeny were grown in a plasticulture production system. We hand-pollinated all flowers on plants at UPitt with pollen from unrelated individuals three times per week, whereas those at USDA-ARS were open pollinated.

\section{Qualitative sex scoring}

Sex phenotype was scored at least twice during AprilMay 2007 at UPitt and May-June 2007 at USDA-ARS. Given the subjective nature of scoring sex as three phenotypic genders (female, male and hermaphrodite), we scored male and female function separately. We scored male function qualitatively based on anther characteristics of 4-8 flowers per plant. Individuals were scored as 'male sterile' when they produced small, white vestigial stamens that lacked pollen-filled anther sacs. When visual scoring of this trait was in question, excised anther sacs were inspected under a compound microscope for the presence of pollen-these individuals never produced pollen. Individuals were scored as 'male fertile' when they produced plump, yellow, pollen-filled anthers. We scored female function qualitatively based on the proportion of flowers that set fruit. To determine fruit set, we enumerated all flowers and fruit for each plant grown in the greenhouse and estimated fruit set as number of fruits divided by number of flowers produced. Plants grown in the field were much larger than those in the greenhouse, and consequently, we were unable to accurately record all flowers produced. Therefore, for field-grown plants, we estimated fruit set based on number of fruits on a plant and an estimate of the number of spent flowers at a given time point.

To facilitate mapping female function as a qualitative trait, we distinguished two classes of female function. We scored plants as 'female sterile' when they set $<5 \%$ of their flowers into fruits and 'female fertile' when they set $\geqslant 5 \%$ of their flowers into fruits. Although this is admittedly a somewhat arbitrary categorization of a continuous trait, we view this as a reasonable first attempt at identifying the region in the genome with a major influence on female function. Moreover, this categorization allowed us to score female function in both parents and to map it as a qualitative trait that segregated in a Mendelian manner (3:1) in the progeny (see below). Male function scores were the same between growth environments for all genotypes. Female function scores between these environments were highly correlated ( $\rho=0.80, P<0.0001)$. For female function, a single consensus score was used such that individuals were considered female sterile only if fruit set was $<5 \%$ in both environments.

\section{DNA extraction}

We extracted genomic DNA from young leaves of greenhouse-grown parents and progeny using a DNeasy Plant Maxi Kit (Qiagen Inc., Valencia, CA, USA). We quantified extracted DNA using the Spectramax 190 spectrophotometer (Molecular Devices, Sunnyvale, CA, USA) and diluted it to $30 \mathrm{ng} \mu \mathrm{l}^{-1}$ with $1 \times$ TE buffer and

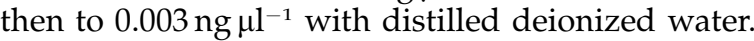

\section{Sources of SSR markers}

Both published and unpublished SSR primer pairs were used in this study. A total of 29 primer pairs from various published sources were tested (Table 1). In addition, 331 new SSR primer pairs (Table 1 and Supplementary Table 1) were developed from publicly available strawberry sequences downloaded from NCBI dbEST. SSR regions were identified by using the CUGISSR.pl script, a modified version of SSRIT (Jung et al., 2005) and filtered for optimal primer development (40-60\% GC content and for presence of at least $20 \mathrm{bp}$ of sequence either side of the motif). Primers were designed from the flanking sequences using Primer3 (Rozen and Skaletsky, 2000). In addition, strawberry sequences were assembled with peach and other Rosaceae EST sequences using CAP3 (Huang and Madan, 1999). The assembled contig sequences were searched for SSR regions, and primer

Table 1 SSR sources

\begin{tabular}{|c|c|c|c|}
\hline Source species and sequence derivation & Total primer pairs tested & Previously published & Primers that amplified polymorphic products \\
\hline F. $\times$ ananassa $\mathrm{EST}$ & 178 & & 106 \\
\hline F. vesca EST & 121 & & 36 \\
\hline F. $\times$ ananassa genomic & 24 & $24^{\mathrm{a}}$ & 20 \\
\hline Rosaceous EST contigs & 28 & & 6 \\
\hline Conserved plant gene sequence & $4^{\mathrm{b}}$ & & 2 \\
\hline F. virginiana genomic & 1 & $1^{\mathrm{c}}$ & 1 \\
\hline F. vesca SCAR & 3 & $3^{\mathrm{d}}$ & 1 \\
\hline F. $\times$ ananassa SCAR & 1 & $1^{\mathrm{e}}$ & 0 \\
\hline Total & 360 & 29 & 172 \\
\hline
\end{tabular}

${ }^{\mathrm{a}}$ Lewers et al. (2005).

${ }^{\mathrm{b}} \mathrm{K}$ Folta and P Stewart (Hort. Sci. Dept. University of Florida), unpublished data.

${ }^{c}$ Ashley et al. (2003).

${ }^{\mathrm{d}}$ Albani et al. (2004).

eHaymes et al. (2000).

SSR primer sources are listed according to source species and sequence derivation. The total number of primer pairs tested is broken down into those that amplified a polymorphic product. Previously published primers are indicated, primers for those previously unpublished see online. 
pairs were developed from the contig sequences to amplify the SSR regions. Primer pair sequences of both the strawberry sequences and the strawberry/peach and strawberry/Rosaceae contigs are publicly available in the Genome Database for Rosaceae (Jung et al., 2008). Primers were also developed from the APETALA3 gene (AY429429) and the CONSTANS gene (AB211134), which was examined in three strawberry cultivars (Table 1).

\section{SSR analysis}

We initially tested all primer pairs on parental DNA. The 184 progeny were scored for polymorphic markers. Parents were tested four times and progeny were tested at least one time for each primer pair that detected polymorphisms. We followed the 'poor man's' PCR protocol described by Schuelke (2000). The PCR reactions for SSR evaluation included $1 \times$ PCR buffer with $1.5 \mathrm{mM}$ $\mathrm{MgCl}_{2}$ (Qiagen Inc.), $0.67 \mathrm{mM}$ dNTPs, $0.4 \mathrm{U}$ HotStar Taq polymerase enzyme (Qiagen Inc.), and template DNA at $0.002 \mathrm{ng} \mathrm{l}^{-1}$ in a total reaction volume of $15 \mu \mathrm{l}$.

We used an MJ Research Engine Tetrad with 96-well blocks (MJ Research, Waltham, MA, USA) for amplification, and heated reaction components to $95^{\circ} \mathrm{C}$ for $15 \mathrm{~min}$ to activate the polymerase, followed by 30 cycles of $40 \mathrm{~s}$ at $94{ }^{\circ} \mathrm{C}, 40 \mathrm{~s}$ at $59^{\circ} \mathrm{C}$ and $40 \mathrm{~s}$ at $72{ }^{\circ} \mathrm{C}$. These first 30 cycles used an annealing temperature $\left(59^{\circ} \mathrm{C}\right)$ specific to the genomic target sequence, whereas the final eight cycles used a lower annealing temperature $\left(52{ }^{\circ} \mathrm{C}\right)$ specific to the M13 sequence to incorporate the fluorescently labeled M13 primer: $40 \mathrm{~s}$ at $94{ }^{\circ} \mathrm{C}, 40 \mathrm{~s}$ at $52^{\circ} \mathrm{C}$ and $40 \mathrm{~s}$ at $72^{\circ} \mathrm{C}$. Reactions were finished with a $10 \mathrm{~min}$ extension period at $72{ }^{\circ} \mathrm{C}$ and storage at $4{ }^{\circ} \mathrm{C}$.

To determine if the reactions resulted in a product, $5 \mu \mathrm{l}$ samples of the PCR products were diluted with $10 \mu \mathrm{l}$ of water and visualized after $12 \mathrm{~min}$ of electrophoresis through a $2 \%$ agarose gel using the E-gel 96 system and software (Invitrogen Corp., Carlsbad, CA, USA). To determine the sizes of the reaction products, $1-2 \mu 1$ samples of the fluorescently labeled PCR products were analyzed using an ABI 3730 DNA Genetic Analyzer and GeneMapper software (Applied Biosystems, Foster City, CA, USA).

\section{Segregation analysis and map construction}

In spite of its disomic inheritance, $F$. virginana is an octoploid, and thus primers might amplify products on more than one chromosome. Therefore, until assumptions of co-dominance can be verified, we chose a conservative strategy of scoring each amplified primer product as a dominant marker. We subsequently performed $\chi^{2}$ goodness-of-fit tests for each marker, including the two sex traits, to determine whether they segregated according to expected Mendelian segregation ratios of either $1: 1$ or $3: 1$. Because of the large number of tests carried out, we only included markers when they fit either a $1: 1$ or $3: 1$ ratio at $P \geqslant 0.0001$, although for the majority of these markers $P$-values were greater than 0.01 (85\% 1:1, 65\% 3:1). For markers segregating 1:1, we identified whether the product originated from the maternal or paternal parent.

We created separate maternal and paternal parent maps in JoinMap 4.0 (Kyazma BV, Wageningen, Netherlands, Van Ooijen, 2006), according to a pseudotest cross strategy (Grattapaglia and Sederoff, 1994). The map for each parent included the specified parent 1:1 markers and all 3:1 markers. Each data set (maternal/ paternal) was treated as a 'backcross' (BC1) population type in JoinMap. For both data sets, we excluded any markers missing data for greater than $25 \%$ of the 184 individuals in the mapping population, as well as individuals missing genetic data for greater than $25 \%$ of the markers. Using independence LOD scores and the default settings in JoinMap, we constructed grouping trees and selected linkage groups at a strict LOD threshold of 10 . To be conservative, groups consisting only of markers originating from the same primer pair were not selected because they may not represent a true linkage group if they cosegregate. We then assigned ungrouped markers to established groups according to the 'Strongest Cross Link' (SCL) values given in JoinMap, using a threshold LOD $\geqslant 4$. We added markers sequentially to avoid erroneous assignments, beginning with the strongest cross link (Van Ooijen, 2006). Subsequently, we created maps for all linkage groups using JoinMap default settings (including the regression mapping algorithm (Stam, 1993) and Haldane's mapping function). In several cases, a set of markers within a linkage group did not map because of 'insufficient linkage' to the rest of the group. This may be caused by repulsion linkage to the remaining markers within the group; we therefore used 'dummy' markers by substituting reversed scores in place of the original data for the markers having insufficient linkage (for example, Mehlenbacher et al., 2006).

We then created a combined map that included both parental sets of 1:1 markers and 3:1 markers. We treated this data set as a 'cross pollination' population type (CP) and allowed linkage phases to be determined by JoinMap. Markers and individuals missing data were excluded using the same criteria as above. We again created groupings as described above. Once these were assigned, we joined linkage groups when possible using SCL values; when two linkage groups contained markers with reciprocal SCL at $\mathrm{LOD} \geqslant 4$, we attempted to join them. In addition, we split linkage groups apart in a few cases when attempts to map markers within a linkage group were unsuccessful and two defined, reliable groups could be established. In cases where multiple mapping rounds were necessary, we examined $\chi^{2}$ values for each marker in the linkage group in the third round and removed markers with an arbitrarily established value of $\chi^{2} \geqslant 4$ in a stepwise manner, beginning with the marker with the highest $\chi^{2}$, until this threshold condition was met for all markers in the linkage group. We also removed markers located $\geqslant 40 \mathrm{cM}$ from another marker; we kept markers located between 30 and $39 \mathrm{~cm}$ from other markers if they linked to at least one marker in the linkage group at LOD $\geqslant 5$. Lastly, we reevaluated agreement with expected segregation ratios for mapped markers using $\chi^{2}$ tests performed by JoinMap to identify potential regions of skewed segregation. Markers were considered to have significantly skewed ratios at $P \leqslant 0.05$.

Relatively few linkage groups in the combined map contained both paternal and maternal 1:1 markers (see Results), such that the combined map not differ greatly from a union of the separate parent maps. Therefore, we present results from the combined map only. Graphic maps were generated using MAPCHART (Voorrips, 2002). 
Genetic map length, map coverage and marker distribution

We estimated the genetic map length, $L$, of $F$. virginiana in two ways. First, we estimated the length of each linkage group $i$ as $\mathrm{G}_{i}=\mathrm{M}_{i}+2 s$, where $\mathrm{M}_{i}$ is the distance between the terminal markers of linkage group $i$ and $s$ is the average marker spacing (Remington et al., 1999; Fishman et al., 2001). Average marker spacing was calculated as the summed length of all linkage groups divided by the total number of marker intervals in the map. The total genetic map length, $L$, was then calculated as $\Sigma G_{i}$ for all $i$ (Fishman et al., 2001). Second, we calculated $L$ as $\Sigma \mathrm{M}_{i}\left(\mathrm{~m}_{i}+1\right) /\left(\mathrm{m}_{i}-1\right)$ for all $i$, where $\mathrm{M}_{i}$ is as before and $\mathrm{m}_{i}$ is the number of markers on linkage group $i$ (Chakravarti et al., 1991). These two methods produced nearly identical estimates of $L$ for our map $(<100 \mathrm{cM}$ difference); therefore, we only present results from the second method. We calculated the map coverage as the proportion of the genome, $c$, that is within 10 and $20 \mathrm{cM}$ of a marker, as in Lange and Boehnke (1982).

We further evaluated whether markers were distributed randomly among linkage groups according to expectations under the Poisson distribution (Remington et al., 1999). The expected number of markers in each linkage group according to a Poisson distribution will be $\lambda_{i}=m \mathrm{G}_{i} / \Sigma \mathrm{G}_{i}$, where $\mathrm{G}_{i}$ is as above and $m$ is the total number of markers in the map. We calculated $\lambda_{i}$ for each linkage group and evaluated the probabilities $P\left(\mathrm{~m}_{i} \leqslant \lambda_{i}\right)$ and $P\left(\mathrm{~m}_{i} \geqslant \lambda_{i}\right)$ under the cumulative Poisson distribution.

\section{Results}

\section{Sex expression}

In our mapping population $(N=184), 90$ progeny were male sterile and 94 were male fertile, whereas 135 of progeny were female fertile and 49 were female sterile as defined here. Thus, when scored qualitatively, male function segregated 1:1 $\left(\chi^{2}=0.04, P=0.77\right)$ and female function segregated $3: 1\left(\chi^{2}=0.26, P=0.61\right)$. Two progeny were both male sterile and female sterile, that is, neuter.

\section{SSR amplification and polymorphism}

Of the 360 total SSR primer pairs tested, 48\% (172) amplified polymorphic products (Table 1). The greatest sources of this latter class were EST-derived F. $\times$ annanassa SSRs $(62 \%)$ and EST-derived F. vesca SSRs (21\%). Each primer pair yielded between 1 and 12 markers, and together the 172 primer pairs yielded 709 markers. From the $\chi^{2}$ tests, we determined that, of these 709 markers plus the two sex traits, 352 approximately fit expected Mendelian segregation ratios of either $1: 1$ or 3:1 according to our initial criteria; 246 segregated 1:1 (111 from the maternal parent; 135 from the paternal parent), and 106 segregated 3:1. These 352 markers were used in map construction.

\section{Genetic map}

The final F. virginiana map includes 210 SSR markers plus the two sex traits and consists of 42 linkage groups (LG) (Figure 1). This is 14 more linkage groups than the expected 28, given the ploidy level and disomic mode of inheritance of this species. The 210 SSR markers were derived from 100 primers, 78 of which were previously unpublished (Supplementary Table 1). In total, including the two sex traits, 80 (38\%) of the 212 markers came from the maternal parent $(72 \%$ of 111 maternal markers included), 93 (44\%) originated from the paternal parent (69\% of 135 paternal included) and 39 (18\%) came from both parents (37\% of 106, 3:1 markers included). Maternal and paternal 1:1 markers were not well integrated among the 42 linkage groups: 16 (38\%) of the linkage groups did not include any 1:1 paternally inherited markers and $21(50 \%)$ did not include any 1:1 maternally inherited markers. Only five (12\%) linkage groups included both of these marker types. However, 28 linkage groups contained at least one shared, 3:1 marker.

Interestingly, the two sex expression traits mapped together on LG 41 (Figure 1), but were separated by $\sim 6 \mathrm{cM}$. This linkage holds when a $10 \%$ threshold for female fertility is used as well (data not shown). Two SSR markers (ARSFL7_277 and ARSFL7_275) mapped to this linkage group. Male sterility was associated with ARSFL7_277 99\% of the time, whereas male fertility was associated with ARSFL7_275 95\% of the time. Female fertility was associated with ARSFL7_277 66\% of the time, and female sterility was associated with ARSFL7_275 88\% of the time. Thus, male sterility, female fertility and ARSFL7_277 are linked in coupling, and all three are linked in repulsion with ARSFL7 275.

Examination of segregation ratios in JoinMap revealed that $64(30 \%)$ of the 212 mapped markers did not fit the expected Mendelian ratios, that is, they showed significantly skewed segregation. These markers were not distributed evenly between paternal and maternal 1:1 markers; a higher percentage of paternal markers exhibited skewed segregation (38\% of paternal markers compared to $13 \%$ of maternal ones). Approximately half of all skewed markers (34) occurred at an end of a linkage group (Figure 1). None of the markers on LG 41 showed unusual segregation.

\section{Map length, map coverage and marker distribution}

Linkage groups ranged in length (that is, the distance between terminal markers) from $\sim 3$ to $131 \mathrm{cM}$, totaling $2373.0 \mathrm{~cm}$. On average, five markers per linkage group ( \pm 2 s.d.; range 2-14) were found (Table 2), with an average marker spacing across the map, $s$, of $14.0 \mathrm{cM}$. The estimated total genetic map length, $L$, was $3640.7 \mathrm{cM}$. According to this estimate, approximately $70 \%$ of the genome is within $10 \mathrm{~cm}$ of a marker on our map, and approximately $90 \%$ is within $20 \mathrm{cM}$ of a marker. If distributed randomly, the expected number of markers in each linkage group should be $\lambda_{i}=212 \mathrm{G}_{i} / 3545.5$. Results from a two-tailed cumulative Poisson distribution test revealed that LG 13 had more markers than expected; markers were distributed as expected for all other linkage groups (Table 2).

\section{Discussion}

Our phenotypic and genetic mapping results support a model of gender determination with at least two linked loci (or gene regions) with major effects, and the existence of both female and hermaphrodite heterogamety in F. virginiana. Evidence of recombination between the loci suggests that $F$. virginiana represents an example of the earliest form of sex chromosomes in plants. Below, we discuss these points in more detail and compare our 
SSR genetic map of this octoploid strawberry to those published for diploid strawberry.

\section{An SSR map of octoploid $F$. virginiana}

The expected number of linkage groups in F. virginiana is 28. Our map resolved 42 linkage groups. Several factors likely contribute to this excess of linkage groups. First, we may simply need more markers to combine some linkage groups. The number of markers used for map construction could have been greater had we not excluded markers because of extremely skewed distributions or had we used less strict LOD thresholds in creating linkage groups. Such measures may have allowed a portion of the 140 ungrouped markers to enter the map and, together, have facilitated joining of linkage groups. However, including skewed markers could have caused spurious linkages (Xian-Liang et al., 2006 and references therein), and because this map is the first SSR map for octoploid strawberry, we chose to be conservative. Our strict protocol generates stable linkage groups. Second, linkage groups in our map are
LG1

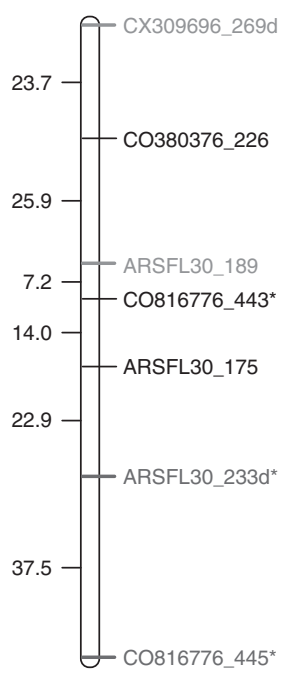

LG5

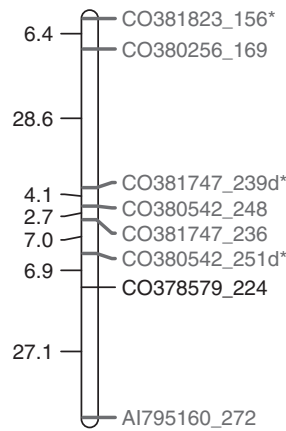

LG9

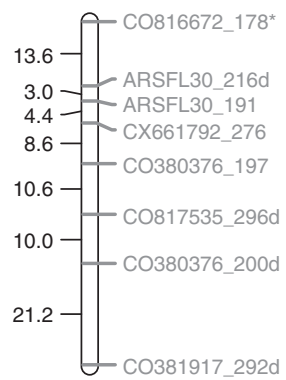

LG2

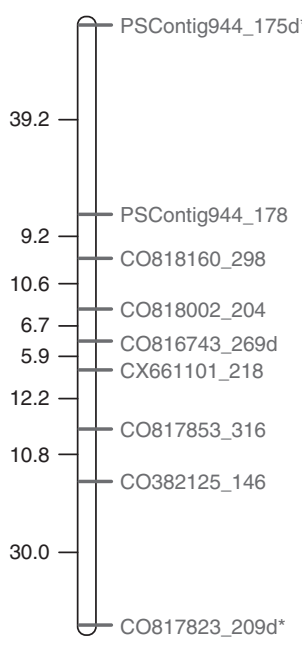

LG6

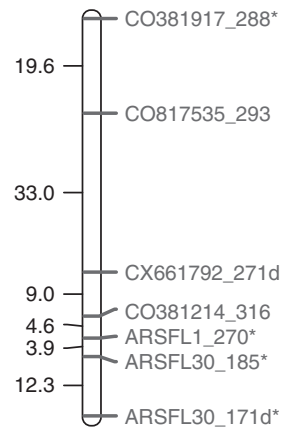

LG10

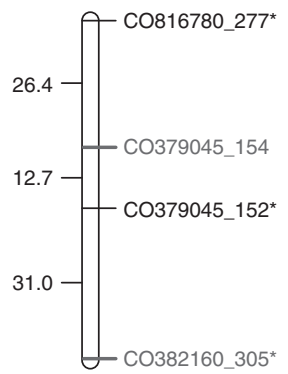

LG3

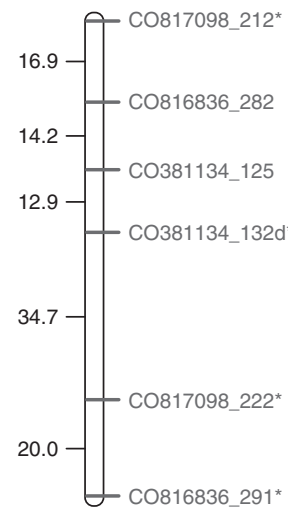

\section{LG4}

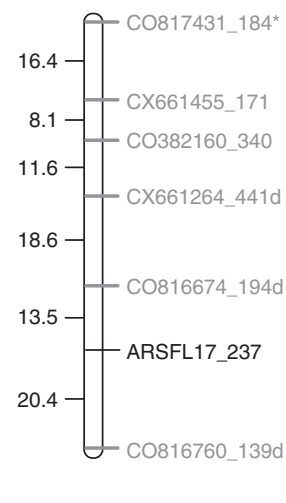

LG7

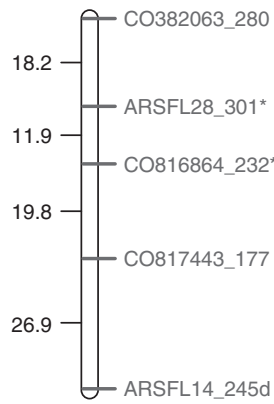

LG11

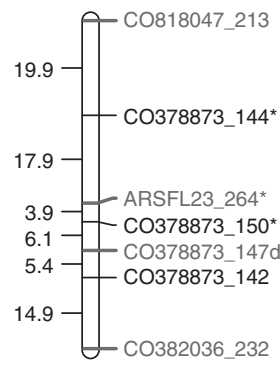

LG8

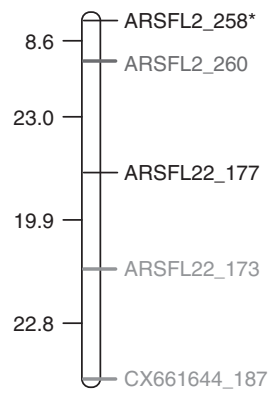

LG12

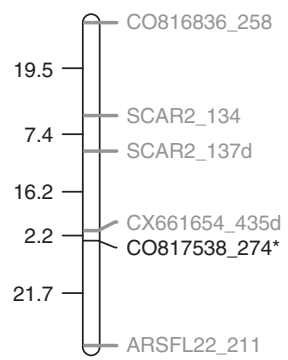

Figure 1 Linkage map of wild strawberry (Fragaria virginiana). Intervals in cM are presented on the left of each linkage group and marker names are listed on the right. Maternally inherited 1:1 markers are coded in red, blue markers represent paternally inherited 1:1 markers, and 3:1 markers are represented in black. Marker names ending in ' $d$ ' identify dummy markers where repulsion linkages are known. Asterisks '*' at the end of marker names identify markers that significantly deviate from expected Mendelian segregation ratios $(P \leqslant 0.05)$. $($ See online version for color figure). 
LG13

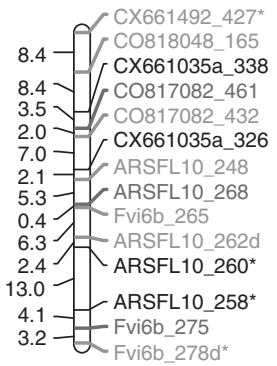

LG17

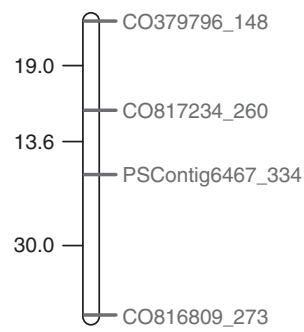

LG21

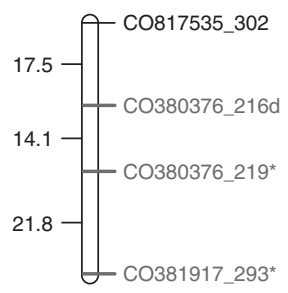

LG25

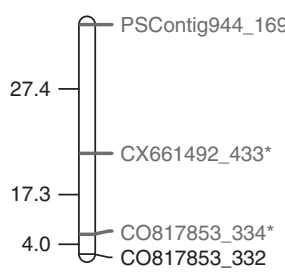

LG14

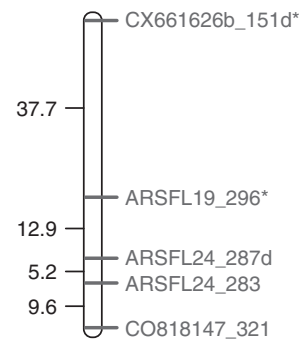

LG18

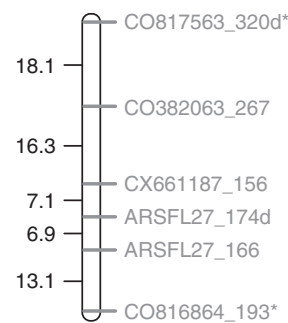

LG22

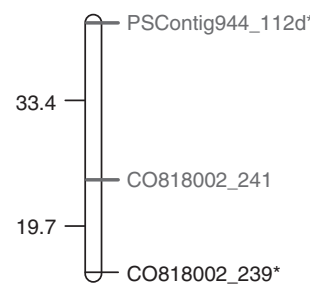

LG26

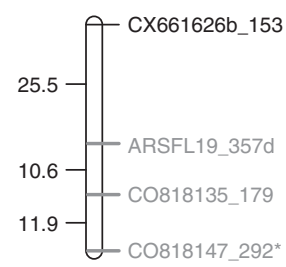

LG15

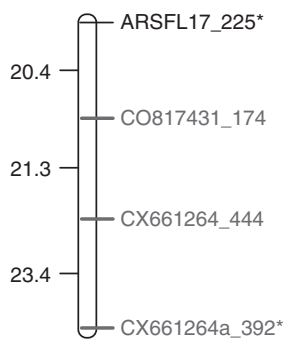

LG19

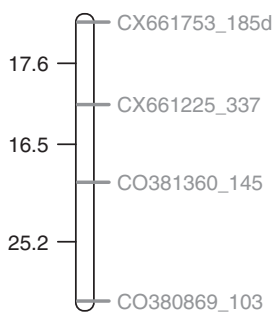

LG23

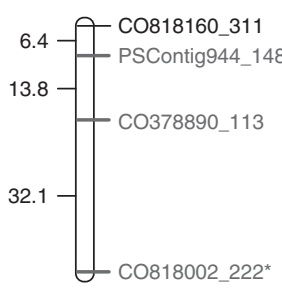

LG27

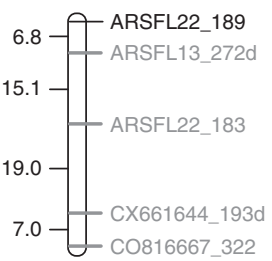

LG16

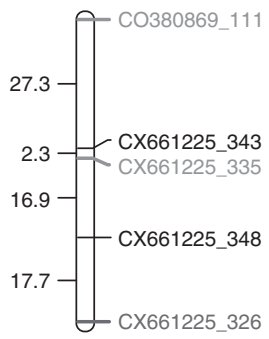

LG20

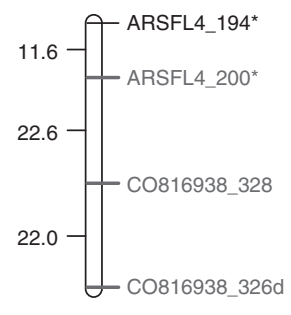

LG24

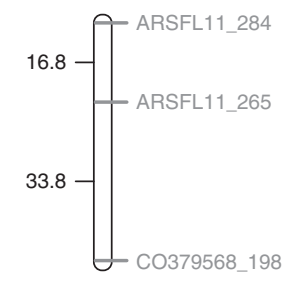

LG28

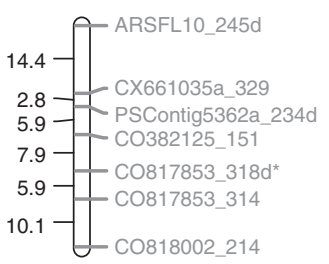

Figure 1 Continued.

noticeably divided into those lacking maternally inherited 1:1 markers and those lacking paternally inherited 1:1 markers. Maps made separately for each parent can potentially be integrated through 3:1 'anchor' markers. Integration and thus reduction of the number of linkage groups may have been impeded by the low number of potential anchor markers on the map in conjunction with relatively low overall marker density, and as more markers are added to the map, we expect several linkage groups to merge with others.

Despite not being fully resolved, our current map covers $\sim 70-90 \%$ of the estimated genome of F. virginiana. Multiple products, or 'alleles', derived from a particular primer pair occur on the same linkage group in many cases, however, and thus we note that the coverage and genome length estimates will change if markers from the same primer pair on a linkage group in fact cosegregate. Separation between product alleles derived from the same primer on a linkage group could be an artifact of genotyping errors; however, separation of these 'alleles' in our map may also be due to changes in a highly heterozygous polyploid genome (Gaeta et al., 2007), necessitating caution. Determination of cosegregation among these 'alleles' in a polyploid genome may be facilitated through the use of new methods such as the use of peak height to determine allele copy numbers in future study (for example, Esselink et al., 2004).

Skewed segregation was apparent in almost a third of the mapped markers. This phenomenon is common and may be due to non-biological sources, such as genotyping and PCR reaction errors (Xian-Liang et al., 2006 and references therein), thus caution must be taken in making interpretations about segregation distortion. For example, a greater proportion of paternal 1:1 markers were skewed than maternal 1:1 markers. Although this could be interpreted as evidence of differential segregation distortion between the sexes, the majority of these 
LG29

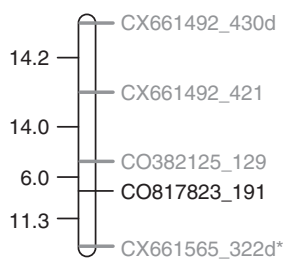

LG32

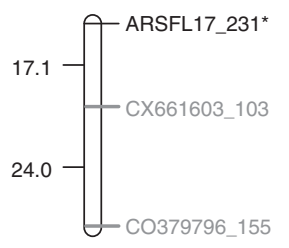

LG37

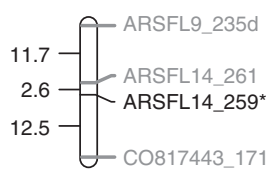

LG41

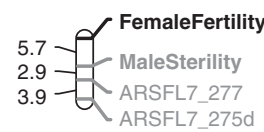

LG30

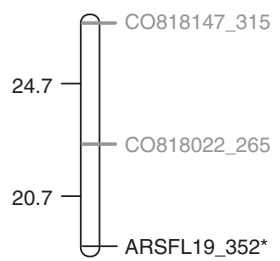

LG34

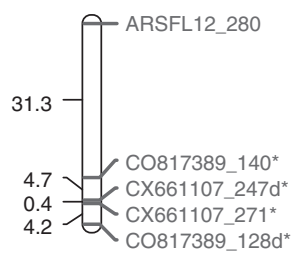

LG38

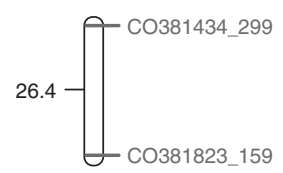

LG42
LG31

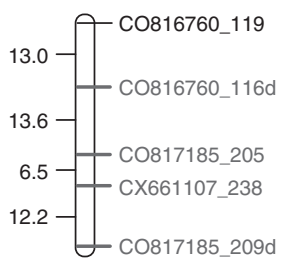

LG35

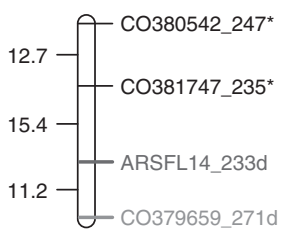

LG39

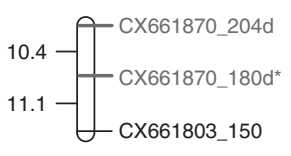

LG32

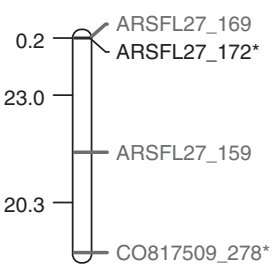

LG36

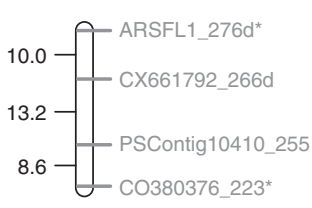

LG40

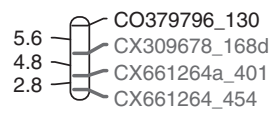

Figure 1 Continued.

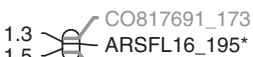

1.3 解 ARSFL16_195*

markers were skewed towards the absence of the marker, suggesting inherent difficulties with PCR amplification rather than segregation distortion. However, we also observed several regions with more than one marker skewed in the same direction. These regions could represent segregation distortion, but this remains to be verified.

\section{Comparison to diploid strawberry map}

Our map represents the first SSR map in an octoploid strawberry. As such, direct comparison between our map and previous maps of octoploid strawberry based on AFLP markers (Lerceteau-Köhler et al., 2003; Weebadde et al., 2008) is difficult. However, previously published diploid strawberry maps (Davis et al., 2006; Sargent et al., 2007) contain SSR markers, 20 of which are in common with our map. Markers derived from the ARSFL9 and ARSFL14 primers mapped to LG 3 in a F. vesca diploid map (Davis et al., 2006), and these two, along with markers from ARSFL28, mapped to LG 3 in two integrated diploid maps (Sargent et al., 2007). In our map, markers from ARSFL9 and ARSFL14 also map together (LG 37) as do markers from ARSFL14 and ARSFL28 (LG 7), indicating some conservation of linkages. In some other cases, unlinked markers in the diploid map are linked in our octoploid map, and vice versa. For instance, our LG 27 contains markers from ARSFL13 and ARSFL22, found on diploid LG 1 and LG 6, respectively (Sargent et al., 2007), and our LG 13 contains markers from ARSFL10 and Fvi6b, also from diploid LG 1 and LG 6, respectively (Sargent et al., 2007). These observations indicate some rearrangements in the octoploid genome between the diploid LG 1 and LG 6 to create the linkage groups present in the octoploid. In addition, the diploid LG 6 contains both ARSFL7 and Fvi6b (Sargent et al., 2007), but these are unlinked on our map. This separation could be due to genome rearrangement and incorporation of the sex loci in the octoploid, but more likely, may be due to the fact that our current map is not completely resolved. Comparisons between diploids and octoploids beyond these are cautioned. As each linkage group in the diploid may be represented by as many as four linkage groups in the octoploid, it is not unlikely that products from the various primer pairs present in one linkage group in the diploid would be represented by more than one linkage group in the octoploid. Likewise, with sequence changes ranging from base substitutions to deletions of various sizes, not all primer pairs with products present in a particular linkage group in the diploid can be expected to be represented in the octoploid.

\section{Sex determination in $F$. virginiana}

Through mapping the two sex functions, our data support a model of sex determination involving at least two linked loci (or gene regions) with major effects. At the male-function locus, a male sterility allele (denoted here as ' $\mathrm{A}$ ' for androecial function) is dominant to an allele conferring male fertility (' $a$ '). At the femalefunction locus, a female fertility allele (' $G$ ' for gynoecial 
Table 2 Marker density and distribution

\begin{tabular}{|c|c|c|c|c|c|}
\hline$L G$ & $m_{i}$ & $M_{i}$ & $G_{i}^{\mathrm{a}}$ & $\lambda_{i}^{\mathrm{b}}$ & $\begin{array}{c}\text { Cumulative } \\
\text { Poisson P-value }\end{array}$ \\
\hline 1 & 7 & 131.2 & 159.1 & 9.5 & 0.269 \\
\hline 2 & 9 & 124.6 & 152.6 & 9.1 & 0.574 \\
\hline 3 & 6 & 98.7 & 126.6 & 7.6 & 0.365 \\
\hline 4 & 7 & 88.6 & 116.5 & 7.0 & 0.599 \\
\hline 5 & 8 & 82.8 & 110.7 & 6.6 & 0.313 \\
\hline 6 & 7 & 82.4 & 110.4 & 6.6 & 0.450 \\
\hline 7 & 5 & 76.8 & 104.7 & 6.3 & 0.399 \\
\hline 8 & 5 & 74.3 & 102.2 & 6.1 & 0.430 \\
\hline 9 & 8 & 71.4 & 99.3 & 5.9 & 0.191 \\
\hline 10 & 4 & 70.1 & 98.0 & 5.9 & 0.299 \\
\hline 11 & 7 & 68.1 & 96.0 & 5.7 & 0.301 \\
\hline 12 & 6 & 67.0 & 94.9 & 5.7 & 0.446 \\
\hline 13 & 14 & 66.1 & 94.0 & 5.6 & 0.006 \\
\hline 14 & 5 & 65.4 & 93.3 & 5.6 & 0.512 \\
\hline 15 & 4 & 65.1 & 93.0 & 5.6 & 0.342 \\
\hline 16 & 5 & 64.2 & 92.1 & 5.5 & 0.529 \\
\hline 17 & 4 & 62.6 & 90.5 & 5.4 & 0.373 \\
\hline 18 & 6 & 61.5 & 89.4 & 5.3 & 0.446 \\
\hline 19 & 4 & 59.3 & 87.2 & 5.2 & 0.406 \\
\hline 20 & 4 & 56.2 & 84.1 & 5.0 & 0.440 \\
\hline 21 & 4 & 53.4 & 81.3 & 4.9 & 0.458 \\
\hline 22 & 3 & 53.1 & 81.0 & 4.8 & 0.294 \\
\hline 23 & 4 & 52.3 & 80.2 & 4.8 & 0.476 \\
\hline 24 & 3 & 50.6 & 78.5 & 4.7 & 0.310 \\
\hline 25 & 4 & 48.7 & 76.6 & 4.6 & 0.513 \\
\hline 26 & 4 & 48.0 & 75.9 & 4.5 & 0.532 \\
\hline 27 & 5 & 47.9 & 75.8 & 4.5 & 0.440 \\
\hline 28 & 7 & 47.0 & 74.9 & 4.5 & 0.173 \\
\hline 29 & 5 & 45.5 & 73.5 & 4.4 & 0.440 \\
\hline 30 & 3 & 45.4 & 73.3 & 4.4 & 0.359 \\
\hline 31 & 5 & 45.3 & 73.3 & 4.4 & 0.440 \\
\hline 32 & 4 & 43.5 & 71.4 & 4.3 & 0.570 \\
\hline 33 & 3 & 41.1 & 69.1 & 4.1 & 0.414 \\
\hline 34 & 5 & 40.6 & 68.5 & 4.1 & 0.440 \\
\hline 35 & 4 & 39.3 & 67.2 & 4.0 & 0.629 \\
\hline 36 & 4 & 31.8 & 59.7 & 3.6 & 0.433 \\
\hline 37 & 4 & 26.8 & 54.7 & 3.3 & 0.433 \\
\hline 38 & 2 & 26.4 & 54.3 & 3.2 & 0.380 \\
\hline 39 & 3 & 21.5 & 49.4 & 3.0 & 0.647 \\
\hline 40 & 4 & 13.2 & 41.2 & 2.5 & 0.238 \\
\hline 41 & 4 & 12.5 & 40.4 & 2.4 & 0.238 \\
\hline 42 & 3 & 2.8 & 30.7 & 1.8 & 0.199 \\
\hline Total & 212 & 2373.1 & 3545.5 & 212 & \\
\hline
\end{tabular}

${ }^{\mathrm{a}} \mathrm{G}_{i}=\mathrm{M}_{i}+2 s$

${ }^{\mathrm{b}} \lambda_{i}=212 \mathrm{G}_{i} / 3545.5$.

For each linkage group (LG) $i$, the observed number of markers $\left(\mathrm{m}_{i}\right)$ observed map length in $c M\left(M_{i}\right)$, inferred map length in $c M\left(G_{i}\right)$, expected number of markers under a Poisson distribution $\left(\lambda_{i}\right)$, and $P$-value associated with the probabilities $P\left(\mathrm{~m}_{i} \leqslant \lambda_{i}\right)$ or $P\left(\mathrm{~m}_{i} \geqslant \lambda_{i}\right)$ under the cumulative Poisson distribution are listed. This is a twotailed test; marker distribution is not significantly different from expected if $P>0.025$

function) is dominant to an allele conferring female sterility (' $\mathrm{g}$ '). On the basis of segregation ratios for the sex function traits in our mapping population and following the two-locus framework, the genotypes for the maternal and paternal parents of our mapping population are hypothesized to be $\mathrm{AaGg}$ and $\mathrm{aaGg}$, respectively (Supplementary Figure 1).

The presence and frequency of neuters as a phenotypic class in our mapping population as well as the creation of hermaphrodites with exceptionally high fruit set (data not shown) are clear evidence of a linked two-locus hypothesis; these classes do not occur under a one-locus model. Recall, neuters were confirmed to be both male and female sterile across six clones per genotype and under hand-outcross pollination in the greenhouse. Furthermore, we have identified neuters and linkage between the two sex function loci in a second map cross involving an interspecific hybrid ( $F$. virginiana and F. chiloensis) (Ashman T-L et al., unpublished data). These recombinant classes are not restricted to our mapping populations; neuter individuals have been found in the wild and in cultivation (Valleau, 1923; Bushakra J, personal communication; Ashman T-L, personal observation), and high-fruiting hermaphrodites have been found in some wild populations of subdioecious F. virginiana (Ashman, 2006) and dioecious F. chiloensis (Hancock and Bringhurst, 1979). The fact that the two sex loci are linked and that recombinants occur at very low frequency, however, may explain why single-locus control was proposed previously (Valleau, 1923; Ahmadi and Bringhurst, 1989). In fact, in our new model, coupling linkage of $G$ and $A$ and of $a$ and $g$ creates chromosomes that correspond to those previously proposed to carry ' $\mathrm{F}$ ' and ' $\mathrm{m}$ ' alleles (Ahmadi and Bringhurst, 1989). Additional confirmation of the twolocus model can be obtained by diagnostic crosses, that is, those that produce phenotypes not possible with the one-locus model. Our preliminary diagnostic test crosses found that the two-locus model better explained the variation in sex expression of the progeny than the onelocus model (unpublished data).

Our two-locus model suggests that for subdioecious F. virginiana, populations will contain females with two genotypes ( $A a G G$ and $A a G g$ ) plus aagg males and $a a G G$ and $A a G g$ hermaphrodites. The frequencies of these genotypes will vary among populations, especially if there is selection against the production of recombinant genotypes (Charlesworth and Charlesworth, 1978). Although linkage between the two sex-determining loci is not an initial requirement for the establishment of subdioecy in a system with dominant female sterility, selection against recombinants will lead to a reduction of recombination between these loci (Charlesworth and Charlesworth, 1978; Bull, 1983). Under continued selection for suppression of recombination between the two loci, subdioecious populations could evolve toward dioecy (Charlesworth and Guttman, 1999). In our system, this process might involve selection for only two types of gametes, that is, those with the sex-function alleles linked in coupling (for example, $A G$ and $a g$ ). This would yield only one genetic configuration for females $(A a G g)$ and one for males, the only pollen-bearing morph (aagg), and lead to the establishment of female heterogametey (Bull, 1983). However, because recombination still occurs between these loci in $F$. virginiana, both females and hermaphrodites are heterogametic. Although dioecy by gynodioecy when female sterility is dominant is thought to be difficult to establish, the sister species to $F$. virginiana,

F. chiloensis, is dioecious. Comparative genomic studies with $F$. chiloensis may thus provide further insight into the evolution of sex determination in Fragaria. Interestingly, although it is easy to see that production of neuter individuals through recombination ought to be selected against, the production of high-fruiting hermaphrodites or the retention of fruiting ability could be maintained in F. virginiana by an autofertility or colonization advantage (Charlesworth, 1999; Ashman, 2006). Thus, ecological 
studies as well as genetic ones will be useful in understanding sex chromosome evolution in this group.

\section{Incipient sex chromosome in strawberry?}

The results presented here point to the intriguing possibility that this linkage group represents an incipient sex chromosome, as linkage between two sex expression loci is an initial step in sex chromosome evolution (Charlesworth et al., 2005). The presence of recombinants and distance between the male and female function loci on our map shows that recombination between the two is not yet suppressed, which is what would be expected in a species with subdioecy (Charlesworth and Guttman, 1999). If this linkage group does represent an evolving sex chromosome, we might expect some of the other recently identified hallmarks of evolving sex chromosomes, that is, low gene density, low cross-species similarity in sex regions and the accumulation of repetitive DNA (for example, Liu et al., 2004; Mariotti et al., 2006; Telgmann-Rauber et al., 2007), as well as traits that show sex-dependent expression and/or sexually antagonistic selection also mapping to the sex linkage group (for example, Scotti and Delph, 2006). The only SSR markers that were associated with the sex region are genomic-derived rather than EST- or sequence-derived, but given the current size of the sex linkage group, current marker density, and excess of linkage groups compared to that expected based on chromosome number, it is too early to evaluate whether this region is indeed gene-poor. We are currently expanding our map to achieve higher marker density, which will allow us to combine some of the 'excess' linkage groups and to assess the relative distribution of EST-derived SSR on the sex linkage group. Moreover, enhancing the density of markers on the sex linkage group in particular, especially with markers that are not derived from expressed genes, and subsequently comparing maternal and paternal maps will inform on the possibility of recombination suppression in the sex region (for example, Liu et al., 2004). Finally, quantitative mapping of sex expression to confirm a single, major region of sex determination in F. virginiana and of traits that show sex-dependent expression (for example, petal area and spring leaf number (Ashman, 2003, 2005)) and sexually antagonistic selection (Ashman, 2005; unpublished data) to determine whether they map to the sex linkage group is an important next step.

\section{Acknowledgements}

We thank D Cole, CL Collin, E Early, J Enns, S Good, E Korns, E Peralta, E Poor, K Rappaport, J Robinson, H Tam, K Williams, L Wright and E York for assistance in the greenhouse, field and laboratory and J van Ooijen and S Mehlenbacher for mapping and statistical advice on JoinMap. We also thank A Telgmann-Rauber, LJ Rowland and A Johnson and anonymous reviewers for comments on a previous version of the manuscript. This project was supported by the USDA Cooperative State Research, Education and Extension Service-National Research Initiative-Plant Genome Program (Grant no. 2005-00765) and NSF DEB-0449488. Mention of trade names or commercial products in this article is solely for the purpose of providing specific information and does not imply recommendation or endorsement by the US
Department of Agriculture. This is contribution 223 to the Pymatuning Laboratory of Ecology.

\section{References}

Ahmadi H, Bringhurst RS (1989). Genetics of sex expression in Fragaria species. Am J Bot 78: 504-514.

Albani MC, Battey NH, Wilkinson MJ (2004). The development of ISSR-derived SCAR markers around the SEASONAL FLOWERING LOCUS (SFL) in Fragaria vesca. Theor Appl Genet 109: 571-579.

Ashley MV, Wilk JA, Styan SMN, Craft KJ, Jones KL, Feldheim JL et al. (2003). High variability and disomic segregation of microsatellites in the octaploid Fragaria virginiana Mill. (Rosaceae). Theor Appl Genet 107: 1201-1207.

Ashman T-L (1999). Determinants of sex allocation in a gynodioecious wild strawberry: implications for the evolution of dioecy and sexual dimorphism. J Evol Biol 12: 648-661.

Ashman T-L (2003). Constraints on the evolution of dioecy and sexual dimorphism: field estimates of quantitative genetic parameters for reproductive traits in three populations of gynodioecious Fragaria virginiana. Evolution 57: 2012-2025.

Ashman T-L (2005). The limits on sexual dimorphism in vegetative traits in a gynodioecious plant. Am Nat 166: S5-S16.

Ashman T-L (2006). The evolution of separate sexes: a focus on the ecological context. In: Harder LD, Barrett SCH (eds). The Ecology and Evolution of Flowers. Oxford University Press: Oxford, UK, pp 204-222.

Bringhurst RS (1990). Cytogenetics and evolution in American Fragaria. HortScience 25: 879-881.

Bull JJ (1983). Evolution of Sex Determining Mechanisms. Benjamin/Cummings: Menlo Park, CA.

Chakravarti A, Lasher LK, Reefer JE (1991). A maximum likelihood method for estimating genome length using genetic linkage data. Genetics 128: 175-182.

Charlesworth B, Charlesworth D (1978). A model for the evolution of dioecy and gynodioecy. Am Nat 112: 975-997.

Charlesworth D (1999). Theories on the evolution of dioecy. In: Geber MA, Dawson TE, Delph LF (eds). Gender and Sexual Dimorphism in Flowering Plants. Springer-Verlag: Berlin, pp 33-60.

Charlesworth D, Guttman DS (1999). The evolution of dioecy and plant sex chromosome systems. In: Ainsworth CC (ed). Sex determination in plants. BIOS Scientific Publishers, LTD: Oxford, pp 25-49.

Charlesworth D, Charlesworth B, Marais G (2005). Steps in the evolution of heteromorphic sex chromosomes. Heredity 95: $118-128$.

Davis TM, DiMeglio LM, Yang R, Styan SMN, Lewers KS (2006). Assessment of SSR marker transfer from the cultivated strawberry to diploid strawberry species: functionality, linkage group assignment, and use in diversity analysis. J Am Soc Hort Sci 131: 506-512.

Esselink GD, Nybom H, Vosman B (2004). Assignment of allelic configuration in polyploids using the MAC-PR (microsatellite DNA allele counting-peak ratios) method. Theor Applied Genet 109: 402-408.

Fishman L, Kelly AJ, Morgan E, Willis JH (2001). A genetic map in the Mimulus guttatus species complex reveals transmission ratio distortion due to heterospecific interactions. Genetics 159: 1701-1716.

Gaeta RT, Pires JC, Iniguez-Luy F, Leon E, Osborn TC (2007). Genomic changes in resynthesized Brassica napus and their effect on gene expression and phenotype. Plant Cell 19: 3403-3417.

Grattapaglia D, Sederoff R (1994). Genetic linkage maps of Eucalyptus grandis and Eucalyptus urophylla using a pseduotestcross: mapping strategy and RAPD Markers. Genetics 137: 1121-1137. 
Hancock JF, Bringhurst RS (1979). Hermaphroditism in predominately dioecious populations of Fragaria chiloensis (L.) Duchn. Bull Torrey Bot Club 106: 229-231.

Haymes KM, Van de Weg WE, Arens P, Maas JL, Vosman B, Den Nijs APM (2000). Development of SCAR markers linked to a Phytophthora fragariae resistance gene and their assessment in European and North American strawberry genotypes. I Am Soc Hort Sci 125: 330-339.

Huang X, Madan A (1999). CAP3: a DNA sequence assembly program. Genome Res 9: 867-877.

Jung S, Abbott A, Jesudurai C, Tomkins J, Main D (2005). Frequency, type, distribution, and annotation of simple sequence repeats in Rosaceae ESTs. Funct Integr Genomics 5: 136-143.

Jung S, Staton M, Lee T, Blenda A, Svancara R, Abbott A et al. (2008). GDR (Genome Database for Rosaceae): integrated web-database for Rosaceae genomics and genetics data. Nucleic Acids Res D1034-D1040 http://www.bioinfo.wsu. edu/gdr.

Lange K, Boehnke M (1982). How many polymorphic genes will it take to span the human genome? Am J Human Genet 24: $842-845$.

Lerceteau-Köhler E, Guérin G, Laigret F, Denoyes-Rothan B (2003). Characterization of mixed disomic and polysomic inheritance in the octoploid strawberry (Fragaria $\times$ ananassa) using AFLP mapping. Theor Applied Genet 107: 619-628.

Lewers KS, Bassil NV, Styan SMN, Hokanson SC (2005). Strawberry GenBank-derived and genomic simple sequence repeat (SSR) markers and their utility with strawberry, blackberry, and red and black raspberry. J Am Soc Hort Sci 130: 102-115.

Liu Z, Moore PH, Ackerman CM, Ragiba M, Yu Q, Pearl HM et al. (2004). A primitive $\mathrm{Y}$ chromosome in papaya marks incipient sex chromosome evolution. Nature 427: 348-352.

Maas JL, Gouin C, Hokanson SC, Hartung JS (2002). Strawberry parent clones US 4808 and US 4809 resistant to bacterial angular leafspot disease caused by Xanthomonas fragariae. HortScience 37: 716-717.

Mariotti B, Navajas-Perez R, Lozano R, Parker JS, de la Herran $\mathrm{R}$, Rejon CR et al. (2006). Cloning and characterization of dispersed repetitive DNA derived from microdissected sex chromosomes of Rumex acetosa. Genome 49: 114-121.

Mehlenbacher SA, Brown RN, Nouhra ER, Gokirmak T, Bassil NV, Kubisiak TL (2006). A genetic linkage map for hazelnut (Corylus avellana L.) based on RAPD and SSR markers. Genome 49: 122-133.

Ming R, Wang J, Moore PH, Paterson AH (2007). Sex chromosomes in flowering plants. Am J Bot 94: 141-150.

Potter D, Luby JJ, Harrison RE (2000). Phylogenetic relationships among species of Fragaria (Rosaceae) inferred from non-coding nuclear and chloroplast DNA sequences. Syst Bot 25: 337-348.

Remington DL, Whetten RW, Liu BH, O'Malley DM (1999). Construction of an AFLP genetic map with nearly complete genome coverage in Pinus taeda. Theor Appl Genet 98: 1279-1292.
Renner SS, Ricklefs RE (1995). Dioecy and its correlates in the flowering plants. Am J Bot 82: 596-606.

Rice WR (1984). Sex chromosomes and the evolution of sexual dimorphism. Evolution 38: 735-742.

Rozen S, Skaletsky HJ (2000). Primer3 on the WWW for general users and for biologist programmers. In: Krawetz S, Misener S, Totowa NJ (eds). Bioinformatics Methods and Protocols. Humana Press: Totowa, NJ pp 365-386.

Sargent DJ, Rys A, Nier S, Simpson DW, Tobutt KR (2007). The development and mapping of functional markers in Fragaria and their transferability and potential for mapping in other genera. Theor Appl Genet 114: 373-384.

Schuelke M (2000). An economic method for the fluorescent labeling of PCR fragments: a poor man's approach to genotyping for research and high-throughput diagnostics. Nature Biotech 18: 233-234.

Scotti I, Delph LF (2006). Selective trade-offs and sex chromosome evolution in Silene latifolia. Evolution 60: 1793-1800.

Stahler MM, Ascher PD, Luby JJ, Roelfs AR (1995). Sexual composition of populations of Fragaria virginiana (Rosaceae) collected from Minnesota and western Wisconsin. Can J Bot 73: $1457-1463$.

Stam P (1993). Construction of integrated genetic linkage maps by means of a new computer package: JoinMap. Plant J 3: 739-744.

Staudt G (1967). The genetics and evolution of heteroecy in the genus Fragaria. I. Investigations on Fragaria orientalis. Z Pflanzenzuchtung 58: 245-277.

Telgmann-Rauber A, Jamsari A, Kinney MS, Pires JC, Jung C (2007). Genetic and physical maps around the sex-determining $M$-locus of the dioecious plant asparagus. Mol Genet Genom 278: 221-234.

Valleau WD (1923). The inheritance of flower types and fertility in the strawberry. Am J Bot 10: 259-274.

Van Ooijen JW (2006). JoinMap 4, Software for the calculation of genetic linkage maps in experimental populations. Kyazma BV: Wageningen, Netherlands.

Voorrips RE (2002). MapChart: software for the graphical presentation of linkage maps and QTLs. J Hered 93 77-78.

Vyskot B, Hobza R (2004). Gender in plants: sex chromosomes are emerging from the fog. Trends Genet 20: 432-438.

Weebadde CK, Wang D, Finn CE, Lewers KS, Luby JJ Bushakra J et al. (2008). Using a linkage mapping approach to identify QTL for day-neutrality in the octoploid strawberry (Fragaria $\times$ ananassa Duch ex Rozier). Plant Breed 127: 94-101.

Xian-Liang S, Xue-Zhen S, Tian-Zhen Z (2006). Segregation distortion and its effect on genetic mapping in plants. Ch J Ag Biotech 3: 163-169.

Yin T, DiFazio SP, Gunter LE, Zhang X, Sewell MM, Woolbright SA et al. (2008). Genome structure and emerging evidence of an incipient sex chromosome in Populus. Genome Res 18: 422-430.

Supplementary Information accompanies the paper on Heredity website (http://www.nature.com/hdy) 